\title{
MANEJ O LAPAROSCÓPICO DE DISPOSITIVO INTRAUTERINO MIGRADO A TROMPA DE FALOPIO CON HIDROSALPINX SECUNDARIO
}

\author{
Jaime Zarhi T.1, Juan Enrique Schwarze M.², Fernando Vacca G. ${ }^{1}$, Johnny Jarzún J. ${ }^{1}$, \\ Fernando Montecinos $R^{1}$
}

${ }_{1}$ Servicio de Obstetricia y Ginecología, Unidad de Cirugía Laparoscopica, Hospital El Pino, San Bernardo. ${ }^{2}$ Unidad de Medicina Reproductiva, Clínica Las Condes.

\section{RESUMEN}

Se presenta el caso clínico de un dispositivo intrauterino ubicado en el lumen de la trompa de Falopio con hidrosalpinx secundario, diagnosticado en forma incidental al realizar una radiografía de columna lumbar, estudio complementado con ecotomografía transvaginal. Se realizó laparoscopia diagnóstica y salpingectomía sin complicaciones.

\section{PALABRAS CLAVES: DIU intraabdominal, laparoscopia}

\section{SUMMARY}

We present a case of asymptomatic IUD inserted in the right uterine tube, with secondary hydrosalpinx. The IUD was found during a non-related X-ray examination of lumbar spine. She underwent laparoscopic surgery and removal of the uterine tube, without any further complication. We discuss the current treatment of intraabdominal IUDs.

\section{KEY WORDS: Intraabdominal IUD, laparoscopy}

\section{INTRODUCCIÓN}

Los dispositivos intrauterinos (DIU) son un método de control de la fertilidad reversible ampliamente usado y altamente efectivo. Cerca de 100 millones de mujeres en el mundo utilizan DIU para controlar su fertilidad y Chile es uno de los países latinoamericanos con la mayor proporción de usuarias de DIU, estimándose que cerca de un $70 \%$ de las usuarias son controladas en los servicios estatales de planificación familiar $(1,2,3)$.

En la actualidad, los más usados son los dispositivos con forma de $T$ medicados con cobre o gestágenos. A diferencia de los DIU no medicados estos son más eficaces y tienen menos efectos adversos (2).
La perforación uterina es una seria complicación asociada a la inserción de un dispositivo. Esta complicación es rara, con una frecuencia promedio estimada en 1,2 por 1000 inserciones y solo el 0,1 a $0,9 \%$ de las perforaciones se asocian a complicaciones severas, a veces con resultado de muerte como la perforación intestinal (4). Esta complicación muchas veces asintomática, ocurre más frecuentemente en el momento de la inserción, pero también puede ocurrir tardíamente, en caso de quedar parcialmente incrustado en el miometrio, produciéndose la migración del dispositivo a la cavidad abdominal. Una vez en la cavidad pélvica o abdominal, el DIU se ubica con mayor frecuencia en epiplón, recto-sigmoides, peritoneo, vejiga, apéndice y anexos, pero la locali- 
zación dentro del lumen de la trompa de Falopio es muy infrecuente $(1,4,5)$. El tratamiento más aceptado para esta complicación, es la remoción ya sea por vía laparoscópica o por laparotomía, principalmente por el riesgo de adherencias intraabdominales y por el potencial daño de órganos vecinos (6).

Se presenta el manejo de un caso de dispositivo intrauterino ubicado en el lumen de la trompa de Falopio con hidrosalpinx secundario.

\section{CASO CLÍNICO}

Mujer de 44 años a quien se le insertó un dispositivo intrauterino (DIU) tipo $\mathrm{T}$ con cobre en el puerperio inmediato, en el año 1978. Durante los 5 años siguientes ella asistió a controles semestrales con matrona en su consultorio, donde se le informaba que las guías del DIU estaban en su lugar.Posteriormente, al solicitar la extracción del dispositivo por deseo de embarazo, es derivada a su hospital de referencia por no observarse las guías. Se solicita ecotomografía transvaginal que descarta presencia de DIU intracavitario, por lo que se concluye su expulsión. Después de una infertilidad secundaria de 4 años de evolución, la paciente se embaraza espontáneamente realizándose una cesárea electiva en 1989. En junio de 2006, por traumatismo accidental en columna lumbar, se realizó una radiografía de pelvis y columna, que reveló la presencia de un DIU tipo $T$ en posición invertida en abdomen superior izquierdo, por lo que es derivada al Servicio para su manejo (Figura 1).En la unidad se confirmó este hallazgo con ecotomografía transvaginal que demostró ausencia de DIU intrauterino.

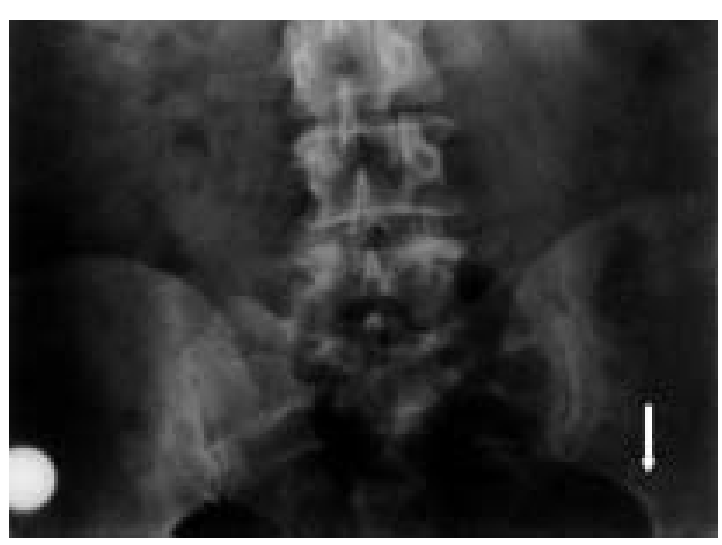

Figura 1. Radiografía de columna lumbar que muestra tenuemente DIU en región pélvica izquierda (flecha).

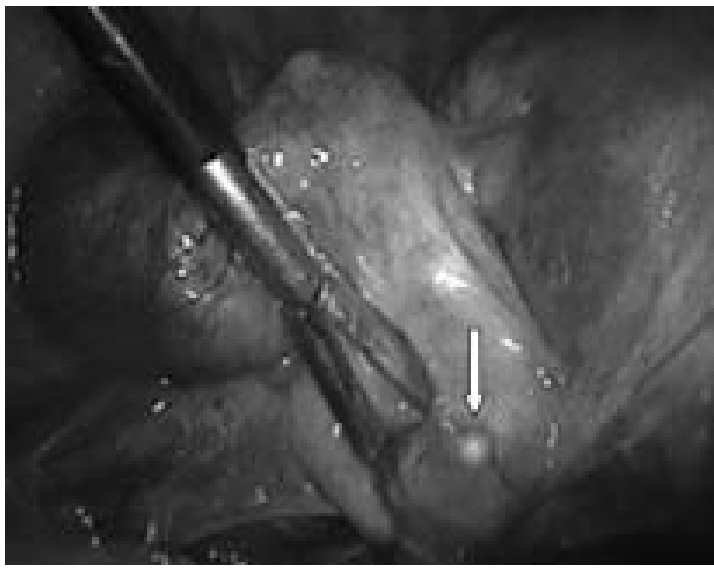

Figura 2. DIU dentro de la trompa izquierda (flecha). Hydrosalpinx y adherencias tubo-ováricas.

El 30 de noviembre de 2006, se realiza laparoscopía diagnóstica, visualizándose hidrosalpinx izquierdo con un DIU tipo T intratubario, sin el filamento de cobre en sus ramas, con mínimas adherencias laxas de trompa a parénquima ovárico (Figuras 2 y 3). No se encontró ninguna solución de continuidad en útero y el anexo derecho se aprecia sin alteraciones. Se procedió a liberación de las adherencias y salpingectomía izquierda con bipolar y tijera. El tiempo operatorio fue de 25 minutos, se usó cefazolina 2 gramos en forma profiláctica y la paciente fue dada de alta en buenas condiciones al día siguiente.

\section{DISCUSIÓN}

Las complicaciones más frecuentes asociada al uso de DIU son las infecciones pélvicas y la

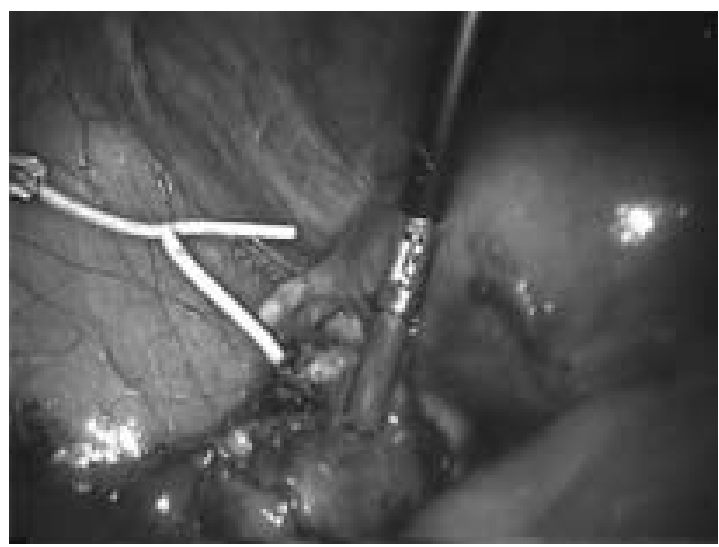

Figura 3. DIU extraído desde trompa izquierda, nótese la ausencia de cobre de las ramas. 
perforación uterina hacia la cavidad pélvica, abdominal y retroperitoneal $(2,5,7)$.

La perforación uterina al momento de la inserción del DIU depende de distintas variables, entre ellas la experiencia del operador, tamaño y posición del útero (anteversión o retroversión), presencia de malformaciones uterinas y periodo de post-parto o post-aborto, que es donde con más frecuencia se produce esta complicación incrementada por una prolongada contractibilidad uterina, acelerada involución y menor dolor durante la inserción en periodo de lactancia $(2,3,8)$. El manejo de una perforación uterina por DIU es contradictorio, ya que la mayoría de las pacientes son asintomáticas y los DIU pueden permanecer silentes por muchos años, diagnosticándose en forma tardía o accidentalmente al realizarse una radiografía de pelvis o columna como ocurrió con esta paciente, a la que se le hizo el diagnóstico después de 28 años. Hay autores que sugieren que la remoción quirúrgica debe realizarse solo en pacientes sintomáticas $(1,6)$.

Los dispositivos medicados con cobre y levonorgestrel, que han perforado completamente la pared uterina hacia la cavidad abdominal, deben ser removidos tan pronto como sea posible, por la gran reacción inflamatoria en los órganos vecinos y por la probabilidad de perforación de una víscera hueca. En el caso de DIU con levonorgestrel sus concentraciones séricas son muy altas (10 veces mayor que cuando esta in situ) interfiriendo con la ovulación, por lo que su extracción es mandatoria si la paciente busca fertilidad (9).

No siempre es posible la extracción de un dispositivo por laparoscopia, ya que muchas veces en ausencia de adherencias, el dispositivo permanece oculto bajo el epiplón mayor o incrustado en él, que hace imposible su localización; en esos casos, es mejor realizar una nueva evaluación en lugar de convertir a laparotomía $(5,10)$. En el caso presentado, encontramos una pelvis libre de adherencias, y un hidrosalpinx izquierdo con una adherencia laxa al ovario, que al liberarla permitió la visualización de la rama vertical del DIU, insinuándose a través de la fimbria, lo que permitió su extracción mediante una salpingectomía sin complicaciones. La tasa de remoción laparoscópica exitosa de un dispositivo varía desde 44 a $100 \%$ en publicaciones nacionales e internacionales $(1,5,7)$.

Se sabe que los dispositivos intrauterinos aumentan 3 a 4 veces la incidencia de enfermedad inflamatoria pélvica, lo que podría producir secundariamente un hidrosalpinx (7). En el caso clínico presentado, lo más probable fue que la presencia del DIU intratubario haya causado una reacción inflamatoria local, que llevó secundariamente a la formación de un hidrosalpinx.

Cuando se realiza una laparoscopia para extraer un DIU, en ocasiones se encuentra solo los señuelos visibles que emergen a través de un plastrón, que al ejercer una tracción excesiva puede dañar seriamente las estructuras adyacentes, además de producir sangrado, de ahí que lo importante en estos casos sea realizar una disección cuidadosa siguiendo los señuelos del DIU para evitar daños (9).

El diagnostico de DIU intraabdominal se realiza inicialmente por ecotomografía transvaginal y en caso de ausencia intracavitaria, debe realizarse una radiografía de pelvis para descartar su expulsión por vía vaginal o confirmar su ubicación intraabdominal. Ocasionalmente, es necesario solicitar un TAC pélvico el cual delimita muy bien la configuración anatómica uterina $(10,11)$. En nuestro caso clínico, la pesquisa se hizo a través de radiografía de columna lumbar solicitada por una contusión, que mostró dispositivo tipo $\mathrm{T}$, invertido en el abdomen superior izquierdo, que fue complementada por una ecotransvaginal que certificó la ausencia del DIU intracavitario. En algunas ocasiones al tomar una radiografía en posición vertical, el DIU puede aparecer en la cavidad pélvica y durante la laparoscopía con la paciente en posición de Trendelenburg puede migrar hacia el abdomen superior lo que dificultad muchas veces el hallazgo, por discrepancia con la localización del diagnóstico radiológico (10).

Por último, la migración de un DIU puede ocurrir dentro de la pelvis y abdomen superior, ubicándose más frecuentemente en el epiplón mayor, rectosigmoides, peritoneo, apéndice y muy rara vez a la trompa de Falopio $(5,7)$. Solo hay un caso publicado en la literatura de una salpingectomía derecha por laparoscopia, realizada por un hidrosalpinx, donde como hallazgo se encontró DIU intratubario (11). En nuestro caso clínico, lo más probable es que en el momento de la inserción, el DIU fue insertado en el ostium tubario y mediante el peristaltismo propio de la trompa, terminó en el interior del lumen tubario, con formación de un hidrosalpinx, que en parte explica la subfertilidad de la pareja.

\section{CONCLUSIÓN}

La ausencia de los señuelos del DIU al examen ginecológico, combinada con la ecotomogra- 
fia transvaginal y radiografía de pelvis, son suficientes para realizar el diagnóstico definitivo de un DIU intraabdominal.

Al realizar una laparoscopia diagnóstica para extraer un DIU, en caso de encontrarse con un plastrón, en que solo son visibles los señuelos, debemos realizar una buena disección hasta ubicar las ramas del DIU, ya que la tracción indebida puede ocasionar serios daños a los órganos adyacentes.

Por último, a pesar de la controversia en relación a si es conveniente la extracción de un DIU intraabdominal, nuestra experiencia (5), nos muestra que por problemas psicológicos un porcentaje muy alto de nuestras pacientes desearon la extracción del DIU a pesar que eran asintomáticas.

\section{BIBLIOGRAFÍA}

1. Shogun MT, Batukan C, Serin IS, Ozcelik B, Basbug $\mathrm{M}$, Dolanbay M. Surgical management of intra-abdominal mislocated intrauterine devices. Contraception 2007;75(2):96-100.

2. Díaz S, Pizarro E. Dispositivos intrauterinos. En: Pérez Sánchez (ed). Editorial Mediterráneo. $3^{\underline{a}}$ edición, 2003; 1074-85.

3. Caliskan E, Oztürk N, Dilbaz BO, Dilbaz S. Analysis of risk factors associated with uterine perforation by intrauterine devices. Eur J Contracept Reprod Health Care 2003;8(3):150-5.
4. Ozdemir H, Mahmutyazicioglu K, Tanriverdi HA, Gündogdu S, Savranlar A, Ozer T. Migration of an intrauterine contraceptive device to the ovary: Case report. J Clin Ultrasound 2004; 32(2):91-4.

5. Zarhi J, Vacca F, Jarzun J, Sepúlveda P, Zapata L. DIU abdomino-pélvico: Manejo laparoscópico. REV Chil Obstet Ginecol 2005, 70(6) 369-374.

6. Marcovitch O, Klein Z, Gidoni Y, Holzinger M, Beyth Y. Extrauterine mislocated IUD: is surgical removal mandatory? Contraception 2002;66(2):105-8.

7. Miranda L, Settembre A, Capasso P, Cuccurullo D, Pisaniello D, Corcione F. Laparoscopic removal of an intraperitoneal translocated intrauterine contraceptive device. Eur J Contracept Reprod Health Care 2003;8(2):122-5.

8. Wynter SH, DaCosta V, Frederick J, Wynter H. Laparoscopic retrieval of perforated intrauterine devices at University Hospital, Jamaica. J Am Assoc Ginecol Laparosc 2002;9(3):380-3.

9. Haimov-Kochman R, Amsalem H, Adoni A, Lavy Y, Spitz IM. Management of a perforated levonorgestrel-medicated intrauterine device: a pharmacokinetic study: Case report. Human Reprod 2003;18(6) 1231-3.

10. Nitke S, Rabineeson D, Dekel A, Sheiner E, Kaplan B, Hackmond R. Lost levonorgestrel DIU: diagnosis and therapy. Contraception 2004; 69:289-93.

11. Sindos M, Pisal N, Setchell M, Singer A. Tubal migration: a rare complication of an intrauterine contraceptive device leading to formation of a hydrosalpinx. Am J Obstet Gynaecol 2003;188(4):1109-10. 